\title{
ON THE FREE PRODUCT OF RINGS WITH WEAK ALGORITHM
}

\author{
R. E. WILliaMS
}

The following theorem is a special case of [1, Proposition 95], a result which requires highly technical methods. The proof we give is elementary.

Let $\left(R_{\lambda}\right)$ denote a family of filtered rings, each with weak algorithm, and for each $\lambda$ let $v_{\lambda}$ be a filtration for which $R_{\lambda}$ satisfies the weak algorithm. By the criterion $\left[2\right.$, p. 335] each $R_{\lambda}$ contains a set $X_{\lambda}$ and a subdivision ring $K_{\lambda}=\left\{a \in R_{\lambda}: v_{\lambda}(a) \leqq 0\right\}$ such that each element has a unique expression

(1) $\sum x_{I} \alpha_{I} \quad\left(\alpha_{I} \in K_{\lambda}\right.$, a.a. $\left.\alpha_{I}=0 ; x_{I}=x_{i_{1}} \cdots x_{i_{n}}, x_{i_{j}} \in X_{\lambda}\right)$.

The crucial part of the proof is establishing a unique form (1) in the free product.

THEOREM. Let $\left(R_{\lambda}\right)$ be a family of filtered rings with weak algorithm as above such that $K$ is the underlying division ring of each $R_{\lambda}$. Then the free product $P$ of $\left(R_{\lambda}\right)$ over $K$ has a unique filtration extending the filtrations on the factors and $P$ satisfies the weak algorithm for this filtration.

Proof. Letting $X=U X_{\lambda}$, each element of $P$ may be written in the form (1) with $X$ replacing $X_{\lambda}$. Assume

$$
\sum x_{I} \alpha_{I}=\sum x_{J} \beta_{J}
$$

write $x_{I}=x_{I_{1}} \otimes \cdots \otimes x_{I_{t}}$ where $x_{I_{1}} \in R_{\lambda_{1}}, \cdots, x_{I_{t}} \in R_{\lambda_{t}}$ and $\lambda_{1}$ $\neq \cdots \neq \lambda_{\text {l. }}$. First suppose there is only one summand on the left so (2) may be rewritten as

$$
x_{I_{1}} \otimes \cdots \otimes x_{I_{t}} \alpha_{I}=\sum x_{J} \beta_{J} .
$$

Now $\sum x_{J} \beta_{J} \in x_{I_{1}} P$, say $\sum x_{J} \beta_{J}=x_{I_{1}} \sum x_{J}, \beta_{J}$, whence

$$
x_{I_{2}} \otimes \cdots \otimes x_{I_{t}} \alpha_{I}=\sum x_{J^{\prime}} \beta_{J^{\prime}}
$$

as $P$ is an integral domain. For $t=1, x_{I_{1}} \in R_{\lambda_{1}}$ implies the right side of (3) is $x_{I_{1}} \alpha_{I}$ and for $t>1$, (4) provides the induction step so the right side of $(3)$ is $x_{I_{1}} \otimes \cdots \otimes x_{I_{1}} \alpha_{I}$ in each case.

If (2) is arbitrary, by moving some of the summands to the right, (3) is recovered so $x_{I} \alpha_{I}=x_{J} \beta_{J}$ for some $I, J$. It follows that the form (1) is unique.

Received by the editors March 14, 1969. 
A valuation may be defined on $P$ as follows. If $x \in X, x \in X_{\lambda}$ for exactly one $\lambda$ and we let $v(x)=v_{\lambda}(x)$. For $x_{I}=x_{i_{1}} \cdots x_{i_{n}}, x_{i_{j}} \in X$, define $v\left(x_{I}\right)=\sum_{1}^{n} v\left(x_{i_{j}}\right)$ and finally extend $v$ to all of $P$ by defining $v\left(\sum x_{I} \alpha_{I}\right)=\max \left\{v\left(x_{I}\right): \alpha_{I} \neq 0\right\}, v(\alpha)=0$ if $\alpha \in K^{*}$ and $v(0)=-\infty$. It is immediate that $v$ is a filtration of $P$ which uniquely extends each $v_{\lambda}$. The remaining conditions that $P$ satisfy the weak algorithm for $v$ may be easily established by the reader.

ACKNowledgements. The author wishes to thank G. M. Bergman for pointing out the results in [1] and the referee for the present form of the theorem.

\section{REFERENCES}

1. G. M. Bergman, Commuting elements in free algebras and related topics in ring theory, Harvard University, Cambridge, Mass., 1967 (unpublished).

2. P. M. Cohn, Rings with a weak algorithm, Trans. Amer. Math. Soc. 109 (1963), 332-356.

KANSAS State University 